\title{
Mobilité internationale en Europe centrale touristes, commerçants et migrants
}

Traduit de l'anglais par Mathieu Fénart

\section{Allan M. Williams et Vladimír Balá}

\section{(2) OpenEdition}

\section{Journals}

Édition électronique

URL : https://journals.openedition.org/remi/1680

DOI : $10.4000 /$ remi. 1680

ISSN : $1777-5418$

\section{Éditeur}

Université de Poitiers

\section{Édition imprimée}

Date de publication : 1 mars 2002

Pagination : 37-65

ISBN : 2-911627-30-X

ISSN : 0765-0752

\section{Référence électronique}

Allan M. Williams et Vladimír Balá, « Mobilité internationale en Europe centrale touristes,

commerçants et migrants ", Revue européenne des migrations internationales [En ligne], vol. $18-n^{\circ} 1$ ।

2002, mis en ligne le 09 juin 2006, consulté le 14 avril 2022. URL : http://journals.openedition.org/ remi/1680; DOI : https://doi.org/10.4000/remi.1680

Ce document a été généré automatiquement le 14 avril 2022

(c) Université de Poitiers 


\section{Mobilité internationale en Europe centrale touristes, commerçants et migrants}

Traduit de l'anglais par Mathieu Fénart

Allan M. Williams et Vladimír Balá

Migration et mobilité

1 En sciences sociales, le terme de «migration» renvoie de façon générale au changement de résidence d'un individu et/ou d'un groupe social ou ethnique. Il existe de nombreux concepts et théories destinés à expliquer les migrations. Les théories néoclassiques de la migration développées par Adam Smith reposent sur la thèse de la maximisation des profits, qui peut provenir soit de l'emploi (Todaro, 1980), soit des disparités dans les prestations sociales (Freeman, 1986). La théorie du capital humain (Sjaastad, 1962) et les nouvelles économies domestiques (the new households economics, Mincer, 1978, Stark, 1991) peuvent être envisagées comme une variante des conceptions néo-classiques. Les conceptions behaviouristes voient la migration comme le résultat de tensions structurelles et anomiques rencontrées par un individu à l'intérieur d'un système sociétal (Lee, 1966, Hoffmann-Nowotny, 1981 et Bauer, 1995). Les approches structuralistes affirment que l'économie mondiale traverse plusieurs étapes de développement, caractérisées par des structures sociales et des cadres de production différents, et qu'il existe d'importantes disparités entre le centre économique et les périphéries (Storper et Walker, 1983). La théorie du marché dual pose l'existence de deux segments dans le marché du travail des économies développées et semi-développées le marché primaire emploie une main-d'œuvre qualifiée et bien rémunérée, offre des emplois stables avec des possibilités de carrière, tandis que le marché secondaire est caractérisé par des emplois précaires avec de bas salaires, un travail pénible et demande un faible niveau de qualification (Massey, 1984).

2 Le terme de "mobilité » fait référence aux différents types de mouvements de population, y compris ceux de courte durée, tels que le tourisme, les voyages d'affaires, les navettes entre le domicile et le lieu de travail ou d'études, etc. L'amélioration des 
moyens de transport et de communication a cependant brouillé ces distinctions. Les changements dans les environnements social, économique et technologique ont à la fois contribué à intensifier les flux migratoires et à remplacer certaines formes de migration par des déplacements de courte durée. Cependant, les concepts sur la mobilité ne peuvent se fonder sur des théories économiques liées à la mondialisation, car ils englobent un ensemble beaucoup plus large de modalités de déplacement.

3 Nous proposons de construire un concept général de mobilité à partir d'un classement hiérarchisé des besoins des hommes. À un niveau élémentaire se situe un besoin immédiat de sécurité personnelle et de survie. Celui-ci est suivi d'un besoin de nourriture, de vêtements, de logement, etc. Lorsqu'un pays progresse vers des niveaux supérieurs de développement socio-économique, ces deux premières incitations à la migration perdent de leur importance. Des individus désireux d'améliorer leur statut social et économique, et qui pensent ne pas pouvoir le faire au sein de leur propre société, remplacent progressivement les réfugiés et les demandeurs d'asile. L'intensité de ces flux est bien moindre que celle générée par les situations de guerres et de conflits. Avec l'amélioration du niveau de vie et la diminution des disparités interrégionales, le volume de ces flux à tendance à décroître. Par contre le tourisme et les loisirs gagnent en importance. Enfin, les sociétés les plus développées permettent l'établissement d'un type particulier de flux migratoire, engendré par les intérêts personnels d'individus privilégiés. Les frontières entre les différents stades ainsi définis sont flottantes; elles varient selon les pays et même les individus. Le tableau 1 schématise les modèles de migration et de mobilité classés selon les étapes de développement des sociétés.

4 Notre propos n'est pas de mettre à l'épreuve toutes les étapes de développement des modèles de mobilité dans différents environnements sociaux et économiques, mais d'en illustrer certains en nous fondant sur nos enquêtes en Europe centrale dans les premiers pays post-communistes, membres de l'OCDE ${ }^{1}$ les Républiques Tchèque et Slovaque, la Hongrie et la Pologne. Cet espace devint une zone tampon entre les pays de l'Union Européenne et ceux des Balkans et d'Europe de l'est, victimes d'un l'effondrement économique après 1989.

5 Les pays d'Europe centrale en cours de transition ont traversé des stades intermédiaires de développement économique, politique et social au cours des dernières années quatre-vingt-dix. Cette évolution n'a engendré ni réfugiés, ni migration de misère. Les mouvements de population en provenance de ces pays ont été principalement motivés par la recherche d'une amélioration du statut socioéconomique (dans le cas des migrants permanents et temporaires) ou par un intérêt personnel (dans le cas des touristes). Les flux touristiques à l'intérieur de cette région ont largement dépassé les flux de migrants, mais les comportements ont gardé certains traits particuliers qui tiennent à la période de transition, tels que la combinaison des raisons économiques et touristiques de se déplacer. 
Tableau 1 : Migration et mobilités, modèles classés selon les besoins des hommes

\begin{tabular}{|c|c|c|c|}
\hline Besoins des hommes & $\begin{array}{l}\text { Migration et } \\
\text { incitation à la } \\
\text { mobilité }\end{array}$ & $\begin{array}{l}\text { Migration et flux } \\
\text { de mobilité }\end{array}$ & Exemples \\
\hline $\begin{array}{l}\text { Aspirations sociales et } \\
\text { thèmes d'interêt } \\
\text { personnel }\end{array}$ & $\begin{array}{l}\text { Loisirs ; passe-temps ; } \\
\text { amélioration de l'état } \\
\text { de santé ; recherche } \\
\text { d'un autre mode de } \\
\text { vie ; travail et vie } \\
\text { dans des } \\
\text { environnements } \\
\text { internationaux }\end{array}$ & $\begin{array}{l}\text { Migration de retraite, } \\
\text { aide au développement } \\
\text { des pays moins } \\
\text { développés, séjours de } \\
\text { remise en forme; } \\
\text { voyages de longue } \\
\text { durée, tourisme de } \\
\text { loisirs }\end{array}$ & $\begin{array}{l}\text { Retraités britanniques } \\
\text { et allemands achetant } \\
\text { des maisons dans la } \\
\text { région } \\
\text { méditerranéenne ; } \\
\text { jeunes américains à } \\
\text { Prague, Paris ou } \\
\text { Amsterdam; } \\
\text { responsables } \\
\text { d'organisations } \\
\text { internationales ; } \\
\text { tourisme }\end{array}$ \\
\hline $\begin{array}{l}\text { Amélioration du statut } \\
\text { socio-économique }\end{array}$ & $\begin{array}{l}\text { Différences de revenus } \\
\text { entre les régions de } \\
\text { destination et d'origine, } \\
\text { mais également à } \\
\text { l'interieur de la région } \\
\text { d'origine }\end{array}$ & $\begin{array}{l}\text { Migrants écono- } \\
\text { miques dont migrants } \\
\text { temporaires et } \\
\text { migrants hautement } \\
\text { qualifiés ; tourisme } \\
\text { d'achats }\end{array}$ & $\begin{array}{l}\text { Émigration à partir } \\
\text { des pays en transition, } \\
\text { migration de cadres et } \\
\text { spécialistes } \\
\text { internationaux ; } \\
\text { tous types de touristes }\end{array}$ \\
\hline $\begin{array}{l}\text { Manque de nourriture, } \\
\text { de vêtements, de } \\
\text { logements }\end{array}$ & Pauvreté & $\begin{array}{l}\text { Migrants } 6 \text { conomiques } \\
\text { dont migrations } \\
\text { permanentes avec des } \\
\text { déqualifications, } \\
\text { petits commerçants } \\
\text { transfrontaliers }\end{array}$ & $\begin{array}{l}\text { La plupart des flux } \\
\text { d'immigrants à partir } \\
\text { du Tiers Monde et des } \\
\text { pays de la CEI }\end{array}$ \\
\hline $\begin{array}{l}\text { Sécurité et survie } \\
\text { personnelles }\end{array}$ & $\begin{array}{l}\text { Guerre, guerre civile ; } \\
\text { persecution ; } \\
\text { catastrophes naturelles }\end{array}$ & $\begin{array}{l}\text { Réfugiés et } \\
\text { demandeurs d'asile }\end{array}$ & $\begin{array}{l}\text { Réfugiés de guerre du } \\
\text { Kosovo, émigration } \\
\text { des états communistes }\end{array}$ \\
\hline
\end{tabular}

6 Nos travaux sur la mobilité en Europe centrale sont fondés sur différentes enquêtes traitant de la migration de main-d'œuvre, du tourisme et de la mobilité liée au commerce transfrontalier. Nous avons mené ces recherches en Slovaquie, proche voisin de l'Autriche (membre de l'Union Européenne), de la République Tchèque, de la Pologne et de la Hongrie (trois économies de transition au même niveau de développement) et de l'Ukraine (pays se situant à une étape de transition moins avancée). La nature différente des situations de part et d'autre de chaque frontière a permis de dégager des modèles de mobilité contrastés. Cette situation nous a fourni une occasion unique d'étudier différents types de flux entre des économies et des sociétés situées à des stades différents de développement. Par exemple, il n'y avait aucune migration de main-d'œuvre entre la Hongrie et la Slovaquie (à l'exception de quelques cas particuliers), mais il y avait des va-et-vient importants motivés par le tourisme et des achats de biens de consommation. Quant aux Slovaques en Autriche, la main-d'œuvre migrante représentait une part intéressante mais minoritaire du total des échanges. Le tourisme et les voyages d'affaires étaient à l'origine de la plupart des mobilités. Les déplacements des Ukrainiens traversant la frontière slovaque étaient motivés par la recherche de revenus, salaires ou bénéfices commerciaux, alors qu'une partie de la population ukrainienne se situait en dessous du seuil de pauvreté. Nous avons également enquêté aux frontières entre la Slovaquie et la Pologne, et nous avons mené un certain nombre d'entretiens avec des responsables du contrôle des frontières et des professionnels du tourisme. Nous faisons référence à ces derniers dans nos comparaisons avec d'autres situations frontalières.

Migration de travail le rôle des origines sociales et économiques

7 La plupart des théories sur les migrations de travail sont élaborées sur la base des différences de développement économique entre les régions d'origine et les régions 
d'accueil. Les modèles classiques de push/pull dégagent un certain nombre de critères (Zimmermann, 1994), notamment, les niveaux de revenus et l'offre d'emploi. Les différentiels de revenus semblaient constituer un facteur bien plus important que les taux de chômage. Dans une étude sur les frontaliers tchèques travaillant en Allemagne

(Horálek, Kos et Horáková, 1996 17), seuls $6 \%$ des navetteurs étaient sans emploi avant de venir occuper leur poste actuel, mais $65 \%$ considéraient l'emploi qu'ils occupaient dans leur pays comme non-satisfaisant ou sans avenir. Une enquête sur la main-d'œuvre migrante étrangère en Autriche (Fassmann, Kohlbacher et Reeger, 1995 58) a montré que " gagner plus d'argent » était une motivation essentielle pour $97 \%$ de Tchèques comme pour $96 \%$ de Polonais et $93 \%$ de Hongrois, alors que le chômage n'était cité, selon les pays, que dans $35,44,27$ et $21 \%$ des réponses.

8 Le niveau des salaires présentait des différences significatives selon les pays d'Europe centrale. Par exemple, le salaire mensuel moyen était d'environ 300 \$US pour la Pologne, la Hongrie et les Républiques Tchèque et Slovaque, mais seulement de 82 \$US en Ukraine. À l'opposé, en Autriche et en Allemagne, il oscillait entre 2200 et 3300 \$US (CESTAT Bulletin 2000, World Bank, 1999). Ces écarts notables généraient d'importants potentiels de migration de main-d'œuvre entre l'Europe de l'ouest et l'Europe centrale, et entre l'Europe centrale et l'Europe de l'est. En termes relatifs, le rapport entre les revenus entre l'Europe de l'ouest et l'Europe centrale semblait plus élevé que celui entre l'Europe centrale et l'Europe de l'est. Les écarts entre les taux de chômage semblaient par contre moins significatifs. En République Tchèque, par exemple, ils se sont maintenus à un faible niveau tout au long des années quatre-vingt-dix, alors que les flux migratoires étaient importants vers l'Allemagne où le niveau du chômage était plus élevé.

Selon les théories néo-classiques de la maximisation des profits, les flux migratoires de l'Europe centrale vers l'Europe de l'ouest devraient être plus importants, ou du moins similaires, à ceux de l'Europe de l'est vers l'Europe centrale. La migration de maind'œuvre devrait être la composante principale des flux transfrontaliers. De plus, l'importance de l'écart entre les revenus du travail devrait entraîner les individus à accepter une déqualification. Selon la théorie du marché dual du travail, la plupart des migrants des deux échantillons devrait donc se trouver dans le segment secondaire. Selon notre approche du développement par étapes, les migrants de l'Europe centrale devaient être "poussés" par des motivations différentes de celles des migrants de l'Europe de l'est.

10 À partir de ces présupposés théoriques sur la migration de travail en Europe centrale, nous avons mené deux enquêtes en Slovaquie, pays d'origine et de destination de la migration. La première concernait la main-d'œuvre migrante slovaque en Autriche, la seconde s'intéressait à la main-d'œuvre migrante ukrainienne en Slovaquie. Nous avons mis à l'épreuve différentes hypothèses

11 - Les deux échantillons possédaient-ils une structure de population similaire (sexe, âge, études, etc.)?

12 - Les motifs de la migration étaient-ils identiques ou similaires dans les deux échantillons?

13 - Y avait-il des différences ou des similitudes significatives dans les comportements d'épargne et de dépense?

Méthodologie de l'enquête 

1999, et concernait 100 travailleurs migrants slovaques. La seconde fut réalisée à la frontière entre la Slovaquie et l'Ukraine, également à l'automne 1999, et concernait 150 migrants ukrainiens. Tous les migrants ont rempli le questionnaire en face-à-face. La plupart des Slovaques étaient originaires des alentours de Bratislava et de la zone Ouest de la Slovaquie, et travaillaient uniquement en Autriche. Tous les migrants ukrainiens étaient originaires de l'oblast de Zakarpatje, qui dispose d'un bon réseau de transports et de nombreux liens historiques et ethniques avec l'ancienne Tchécoslovaquie. Nous pensions que tous les migrants ukrainiens travailleraient dans les Républiques Tchèque et Slovaque, mais certains se dirigeaient également vers des pays de l'Europe de l'ouest et du sud (Allemagne, Espagne, Italie). Les répondants pouvaient choisir entre plusieurs réponses aux différentes questions, mais la taille de l'échantillon nous a conduits à opérer des regroupements. Les associations les plus significatives sont reportées dans le tableau 3, tandis que le détail des regroupements est présenté dans les notes de ce tableau.

Résultats de l'enquête et $63 \%$ des Ukrainiens étaient âgés de moins de 35 ans. Par contre il existait une différence significative dans la répartition des sexes les Slovaques comptaient $16 \%$ de femmes, contre $42 \%$ chez les Ukrainiens. diversement avec les autres variables dans les deux groupes. Tandis que la plupart des hommes slovaques $(81 \%)$ étaient mariés, les femmes étaient plus fréquemment célibataires (56\%). Chez les Ukrainiens, la situation était différente seuls $54 \%$ des hommes et $46 \%$ des femmes étaient mariés. Comme chez les Slovaques, la variable sexe était corrélée au niveau d'études et au salaire à l'étranger. Les hommes montraient un niveau d'études et des revenus plus élevés que les femmes (tableau 3). Par contre, les femmes ukrainiennes possédaient un niveau d'études similaire à celui des hommes, mais cette donnée n'avait pas d'incidence dans les postes occupés ou les niveaux de revenus. Les femmes ukrainiennes avaient des revenus plus faibles que ceux obtenus dans leur emploi précédent et occupaient également des positions professionnelles inférieures, alors que les hommes réussissaient plus fréquemment à occuper des emplois qualifiés (cadres, professions libérales). Les femmes ukrainiennes n'ont commencé à émigrer pour travailler qu'après 1994, plus tardivement que les hommes.

Chaque groupe comprenait une notable proportion de diplômés $21 \%$ des migrants slovaques et $40 \%$ des ukrainiens déclaraient posséder un diplôme universitaire. Cependant, seuls les Slovaques semblaient réussir à valoriser leur niveau de formation. En effet, il n'y avait pas de relation significative entre le niveau d'études et le nouvel emploi des Ukrainiens alors que pour les Slovaques, la formation déterminait davantage l'emploi obtenu en émigration que celui précédemment occupé en Slovaquie. Un tiers des Slovaques, possédant un diplôme universitaire étaient cadres ou exerçaient une profession libérale en Autriche. En émigration, comme auparavant en Ukraine, les migrants ukrainiens étaient employés dans le secteur du bâtiment et de l'agriculture, ou tenaient un petit commerce (coefficient élevé de corrélation entre l'ancien et le nouveau poste occupé, tableau 3). Le processus de déqualification des diplômés était déjà entamé avant l'émigration. 

long de l'échelle des salaires, les fréquences les plus élevées ( $23 \%$ de l'ensemble) correspondant à un salaire de 20 à 30000 schillings autrichiens (1430-2140 \$US), suivi par des salaires de 860 à 1140 \$US (21\%) et 570 à 860 \$US (20\%). En général, les salaires des migrants slovaques étaient légèrement inférieurs à la moyenne autrichienne, mais ils atteignaient les niveaux autrichiens pour les emplois les plus qualifiés, notamment les cadres. Cette situation bénéficiait aux diplômés, et plus fréquemment aux hommes qu'aux femmes, différence qui n'existait pas dans leurs emplois antérieurs en Slovaquie. Enfin, il est intéressant de constater que le revenu en Autriche était positivement corrélé au niveau du poste occupé avant la migration, plus nettement qu'à celui du poste actuellement occupé en Autriche. Cela pourrait indiquer l'importance que les employeurs autrichiens accordaient à l'expérience professionnelle acquise avant la migration.

19 L'écart entre les salaires en immigration et ceux au pays d'origine était très élevé pour les Ukrainiens travaillant à l'étranger, dépassant les moyennes mesurées par la Banque Mondiale (1999). L'écart le plus fréquent entre le pays d'origine et d'accueil constaté par cet organisme était de 6 à 10, semblable à celui que nous avons constaté pour les Slovaques en Autriche. Les Ukrainiens que nous avons interrogé avaient un salaire moyen en Ukraine inférieur à 25 \$US pour le quart d'entre eux, entre 25 et 50 \$ pour $39 \%$; seuls $14 \%$ déclaraient un salaire supérieur à 100 \$. À l'étranger, leurs revenus ont augmenté de façon spectaculaire. Plus de la moitié d'entre eux ont déclaré des revenus mensuels moyens de 200 à 300 \$US. Cette somme semblait crédible, car elle n'était que légèrement inférieure aux salaires moyens en Tchéquie comme en Slovaquie. Il était intéressant de constater que les migrants ukrainiens travaillant en Europe de l'ouest déclaraient approximativement les mêmes salaires que ceux travaillant dans les pays en cours de transition, ce qui semble confirmer le caractère peu qualifié des emplois qu'ils occupaient.

20 Les Ukrainiens travaillant à l'étranger vivaient avec des budgets serrés la moitié d'entre eux étaient capables d'épargner de 30 à $50 \%$ des revenus, et $20 \%$ épargnait de 50 à $70 \%$. Étant donné le bas niveau général des salaires (entre 200 et 300 \$US) et le coût élevé de la vie dans les Républiques Tchèque et Slovaque, les Ukrainiens vivaient donc de façon spartiate. Les Slovaques en Autriche se permettaient des dépenses plus importantes et une épargne moindre. Un peu moins de la moitié d'entre eux épargnaient de 30 à $50 \%$ de leurs revenus et $40 \%$ en épargnaient moins de $30 \%$.

21 Le motif de l'épargne différait également entre les deux groupes de migrants. Près de la moitié des Slovaques économisait de l'argent afin d'acheter, agrandir et réparer une maison (24 \%) ou un appartement (19\%). Environ un cinquième des migrants utilisaient leur salaire pour couvrir les besoins courants de la famille et $18 \%$ économisaient principalement par précaution. Le but le plus fréquent de l'épargne des Ukrainiens, cité par $55 \%$ d'entre eux, était de couvrir les besoins courants de leur famille (loyer, nourriture, vêtements, etc.). Cette réponse est presque générale chez les femmes, qui n'ont commencé à émigrer qu'après les baisses significatives du niveau de vie. Pour l'ensemble des interviewés, l'achat d'une maison, d'un appartement ou d'un terrain ne représentait que $15 \%$ des réponses, et la réparation d'une maison ou d'un appartement que $7 \%$.

22 La fréquence des déplacements des migrants est fortement liée à des facteurs géographiques. Les soixante kilomètres qui séparent Bratislava de Vienne offrent la 
possibilité aux migrants d'effectuer des navettes quotidiennes ou hebdomadaires (49 et $42 \%$ respectivement) ; la faiblesse de la distance permettait ainsi une vie familiale normale. Les Slovaques mariés, occupant en Autriche des emplois hautement qualifiés ou des postes d'encadrement se trouvaient fréquemment dans ce cas de figure, d'autant plus que, ainsi que l'a constaté Kollár (1998), les migrants de Bratislava ont un niveau d'études et de revenus plus élevés que les migrants des zones rurales. A contrario la plupart des Ukrainiens devaient parcourir plusieurs centaines de kilomètres pour se rendre à Bratislava, Prague et les autres villes européennes. Ainsi $43 \%$ des migrants ne retournaient en Ukraine que quelques fois par an et $9 \%$ encore moins souvent, tandis que seuls $25 \%$ étaient en mesure d'y retourner au moins une fois par mois. Les navetteurs quotidiens et hebdomadaires ne représentaient que 9 et $15 \%$.

Tableau 2 : Salaires nets de la main-d'œuvre migrante slovaque en Autriche et de la main-d'œuvre ukrainienne en Slovaquie (effectifs)

\begin{tabular}{|c|c|c|c|c|}
\hline \multirow{2}{*}{\multicolumn{2}{|c|}{ SLOVAQUES EN AUTRICHE }} & \multicolumn{2}{|c|}{$\begin{array}{l}\text { Quel est votre salaire net moyen } \\
\text { actuel ? }\end{array}$} & \multirow[t]{2}{*}{ Total } \\
\hline & & jusqu'à 1140 \$US & Plus de 1140 \$US & \\
\hline $\begin{array}{l}\text { Lors de votre emploi } \\
\text { précédent en Slovaquie, } \\
\text { vous étiez : }\end{array}$ & $\begin{array}{l}\text { Cadre, entrepreneur ou } \\
\text { profession libérale } \\
\text { Autre catégorie } \\
\text { Total }\end{array}$ & $\begin{array}{r}6 \\
48 \\
54 \\
\end{array}$ & $\begin{array}{l}14 \\
28 \\
42 \\
\end{array}$ & $\begin{array}{l}20 \\
76 \\
96\end{array}$ \\
\hline Sexe & $\begin{array}{l}\text { Homme } \\
\text { Femme } \\
\text { Total }\end{array}$ & $\begin{array}{l}41 \\
13 \\
54\end{array}$ & $\begin{array}{r}40 \\
2 \\
42 \\
\end{array}$ & $\begin{array}{l}81 \\
15 \\
96\end{array}$ \\
\hline Niveau d'études & $\begin{array}{l}\text { Primaire, secondaire } 1^{\text {er }} \\
\text { et } 2^{\text {eme }} \text { cycle } \\
\text { Universitaire } \\
\text { Total }\end{array}$ & $\begin{array}{r}47 \\
7 \\
54\end{array}$ & $\begin{array}{l}29 \\
12 \\
41\end{array}$ & $\begin{array}{l}76 \\
19 \\
95\end{array}$ \\
\hline \multirow{2}{*}{\multicolumn{2}{|c|}{ UKRAINIENS EN SLOVAQUIE }} & \multicolumn{2}{|c|}{$\begin{array}{l}\text { Quel est votre salaire net moyen } \\
\text { actuel ? }\end{array}$} & \multirow[t]{2}{*}{ Total } \\
\hline & & jusqu’à 1140 \$US & Plus de 1140 \$US & \\
\hline $\begin{array}{l}\text { Lors de votre emploi } \\
\text { précédent en Ukraine, } \\
\text { vous étiez : }\end{array}$ & $\begin{array}{l}\text { Cadre, entrepreneur ou } \\
\text { profession libérale } \\
\text { Autre catégorie } \\
\text { Total }\end{array}$ & $\begin{array}{l}18 \\
80 \\
98\end{array}$ & $\begin{array}{r}7 \\
22 \\
29\end{array}$ & $\begin{array}{l}25 \\
102 \\
127\end{array}$ \\
\hline Sexe & $\begin{array}{l}\text { Homme } \\
\text { Femme } \\
\text { Total }\end{array}$ & $\begin{array}{r}61 \\
39 \\
100\end{array}$ & $\begin{array}{r}20 \\
9 \\
29\end{array}$ & $\begin{array}{r}81 \\
48 \\
129 \\
\end{array}$ \\
\hline Niveau d'études & $\begin{array}{l}\text { Primaire, secondaire } 1^{\text {er }} \\
\text { et } 2^{\text {eme }} \text { cycle } \\
\text { Universitaire } \\
\text { Total }\end{array}$ & $\begin{array}{l}59 \\
40 \\
99\end{array}$ & $\begin{array}{r}19 \\
9 \\
28\end{array}$ & $\begin{array}{r}78 \\
49 \\
49 \\
127\end{array}$ \\
\hline
\end{tabular}

Source : enquête des auteurs, 1999

Nos enquêtes ont montré plusieurs similitudes dans les modèles de migration de travail dans la zone tampon de l'Europe centrale, mais aussi des différences significatives. Les origines sociales des deux groupes étaient similaires les migrants slovaques comme les migrants ukrainiens étaient jeunes et instruits, et pouvaient espérer des revenus intéressants, étant donné les importantes différences de salaire entre les pays d'origine et de destination. Cependant, la situation économique était différente dans les pays d'origine. L'Europe centrale avait une position assez ambiguë. Alors qu'elle était une périphérie économique comparée à l'Autriche et l'Allemagne, elle était également un centre économique pour les migrants d'Ukraine. Dans les deux échantillons, la maximisation des profits était la principale raison à l'origine de la migration, comme le suggèrent les théories néo-classiques. Selon la théorie duale du marché, les migrants slovaques et les migrants ukrainiens devaient occuper dans les économies centrales des 
emplois peu payés et demandant peu de qualification. Cependant, ceci n'était que partiellement vrai.

Tableau 3 : Travailleurs migrants slovaques en Autriche et ukrainiens à l'étranger. Coefficients de corrélation

\begin{tabular}{|l|c|c|c|c|c|c|c|}
\hline $\begin{array}{l}\text { SLOVAQUES EN } \\
\text { AUTRICHE }\end{array}$ & Sexe & Âge & Études & $\begin{array}{c}\text { Ancien } \\
\text { salaire }\end{array}$ & $\begin{array}{c}\text { Nouveau } \\
\text { salaire }\end{array}$ & $\begin{array}{c}\text { Ancien } \\
\text { poste }\end{array}$ & $\begin{array}{c}\text { Nouveau } \\
\text { poste }\end{array}$ \\
\hline Sexe & $\mathrm{x}$ & $\mathrm{x}$ & $\mathrm{x}$ & $\mathrm{x}$ & $\mathrm{x}$ & $\mathrm{x}$ & $\mathrm{x}$ \\
\hline Âge & $\mathrm{n}$ & $\mathrm{x}$ & $\mathrm{x}$ & $\mathrm{x}$ & $\mathrm{x}$ & $\mathrm{x}$ & $\mathrm{x}$ \\
\hline Études & 0.322 & $\mathrm{n}$ & $\mathrm{x}$ & $\mathrm{x}$ & $\mathrm{x}$ & $\mathrm{x}$ & $\mathrm{x}$ \\
\hline Ancien salaire & $\mathrm{n}$ & $\mathrm{n}$ & $\mathrm{n}$ & $\mathrm{x}$ & $\mathrm{x}$ & $\mathrm{x}$ & $\mathrm{x}$ \\
\hline Nouveau salaire & 0.255 & $\mathrm{n}$ & 0.198 & $\mathrm{n}$ & $\mathrm{x}$ & $\mathrm{x}$ & $\mathrm{x}$ \\
\hline Ancien poste & $\mathrm{n}$ & $\mathrm{n}$ & 0.210 & $\mathrm{n}$ & 0.262 & $\mathrm{x}$ & $\mathrm{x}$ \\
\hline Nouveau poste & $\mathrm{n}$ & $\mathrm{n}$ & 0.275 & $\mathrm{n}$ & $\mathrm{n}$ & $\mathrm{n}$ & $\mathrm{x}$ \\
\hline $\begin{array}{l}\text { UKRAINIENS EN } \\
\text { SLOVAQUIE }\end{array}$ & $\mathrm{Sexe}$ & $\hat{A} g e$ & Études & Ancien & Nouveau & Ancien & Nouveau \\
poste & poste \\
\hline Sexe & $\mathrm{x}$ & $\mathrm{x}$ & $\mathrm{x}$ & $\mathrm{x}$ & $\mathrm{x}$ & $\mathrm{x}$ & $\mathrm{x}$ \\
\hline Áge & $\mathrm{n}$ & $\mathrm{x}$ & $\mathrm{x}$ & $\mathrm{x}$ & $\mathrm{x}$ & $\mathrm{x}$ & $\mathrm{x}$ \\
\hline Études & $\mathrm{n}$ & $\mathrm{n}$ & $\mathrm{x}$ & $\mathrm{x}$ & $\mathrm{x}$ & $\mathrm{x}$ & $\mathrm{x}$ \\
\hline Ancien salaire & 0.274 & $\mathrm{n}$ & $\mathrm{n}$ & $\mathrm{x}$ & $\mathrm{x}$ & $\mathrm{x}$ & $\mathrm{x}$ \\
\hline Nouveau salaire & $\mathrm{n}$ & $\mathrm{n}$ & $\mathrm{n}$ & 0.185 & $\mathrm{x}$ & $\mathrm{x}$ & $\mathrm{x}$ \\
\hline Ancien poste & 0.258 & $\mathrm{n}$ & $\mathrm{n}$ & $\mathrm{n}$ & $\mathrm{n}$ & $\mathrm{x}$ & $\mathrm{x}$ \\
\hline Nouveau poste & 0.210 & $\mathrm{n}$ & $\mathrm{n}$ & $\mathrm{n}$ & 0.212 & 0.538 & $\mathrm{x}$ \\
\hline
\end{tabular}

Réponses uniques. Seules les corrélations ayant un niveau de signification de 0.05 ont été reportées. $\mathrm{n}=$ non significatif.

Sexe : $1:$ homme, 2 : femme ; âge : $1:$ jusqu'à $34,2: 35$ et plus ; niveau d'éducation : 1 : primaire, secondaire $1^{\text {er }}$ et $2^{\text {eme }}$ cycle, 2 : université ; ancien salaire (salaire lors du dernier emploi) : 1 : jusqu'à 180 \$US, 2 : plus de 180 \$US en Slovaquie : et 1 : jusqu'à $50 \$$ US, 2 : plus de 50 \$US en Ukraine; nouveau salaire (salaire de l'emploi actuel) : 1 : jusqu'à 1140 \$US, 2 : plus de 1140 \$US en Slovaquie et 1 : jusqu’à $300 \$$ US, 2 : plus de 300 \$US en Ukraine ; ancien poste (poste lors du dernier emploi en Slovaquie ou en Ukraine) : $1:$ profession libérale ou cadre, 2 : autre ; nouveau poste (poste dans l'emploi actuel à l'étranger) : 1 : entrepreneur, profession liberale ou cadre, 2 : autre.

Source : enquêtes des auteurs, 1999

Le niveau de vie était significativement plus élevé en Slovaquie qu'en Ukraine. L'intérêt des Slovaques pour le travail à l'étranger était principalement dû à un désir d'amélioration du statut social (avoir un plus bel appartement, acheter une nouvelle voiture, etc.). Beaucoup d'entre eux exerçaient une profession très qualifiée ou occupaient un poste d'encadrement avant la migration et refusaient une déqualification. Les Ukrainiens, par contre, se trouvaient dans une position plus difficile. Ceci était particulièrement vrai pour les jeunes femmes diplômées. D'un côté, la différence relative des revenus entre l'Autriche et la Slovaquie était aussi élevée que la différence entre la Slovaquie et l'Ukraine (de 6 à 10 fois), et de l'autre, le rapport entre les prix et les revenus était plus défavorable en Ukraine, les incitant à émigrer même au prix d'une déqualification.

Le petit commerce transfrontalier. Une forme de migration de travail ?

Le petit commerce transfrontalier repose sur les différentiels de prix, le taux de change et la disponibilité des produits de part et d'autre de la frontière. La frontière est à la fois un obstacle et une opportunité (Thuen, 1999, Wallace et alii, 1996) pour les flux de main-d'œuvre, de biens ou de capitaux. Plus les différences sont grandes, plus les profits peuvent être élevés (Altvater, 1998). Alors que le petit commerce transfrontalier existe à toute frontière, les profits sont d'autant plus élevés que les frontières séparent (et réunissent) deux systèmes socio-économiques contrastés (économies avancées et économies en voie de développement, économies de marché et économies planifiées). 
26 Le petit commerce transfrontalier s'est développé en Europe centrale et de l'Est au moins depuis les années soixante et soixante-dix, dès que les obstacles majeurs à la mobilité entre les pays du Pacte de Varsovie ont été surmontés. Le gouvernement hongrois de Kadar, par exemple, suivait la stratégie du "socialisme goulasch» («tu te tais et je paie ton goulasch»), combinant le bâton de la répression politique et la carotte des biens de consommation importés des pays de l'Ouest. Bien sûr, ces biens de consommation étaient également recherchés par les citoyens des autres pays communistes, qui jouissaient d'une relative liberté de circulation et d'échanges avec la Hongrie. Les biens achetés par les touristes dans des « pays socialistes frères » étaient destinés à la consommation personnelle, et non à la revente. Une grande partie du tourisme dans la région était l'occasion de faire des achats.

Une nouvelle manière de pratiquer le petit commerce international a débuté au début des années quatre-vingts, lorsque le gouvernement polonais s'est trouvé dans l'obligation de libéraliser les échanges, y compris avec les pays de l'Ouest. Des milliers de consommateurs polonais sont devenus marchands, achetant des biens dans les pays de l'Ouest (principalement en Allemagne et en Autriche) et les revendant en Pologne. La suppression du passeport et du visa après 1989 a fortement contribué au développement de ce petit commerce international. La diminution des freins à la mobilité a coïncidé avec une période de profonde récession économique, de montée du chômage et de forte baisse du niveau de vie dans la région. Le petit commerce international est devenu une stratégie de survie économique alternative, source principale ou secondaire de revenus pour de nombreux foyers. Ces phénomènes, liés à l'essor des marchés en plein air et au développement du " commerce à la valise » ont été explorés dans de nombreuses études, en particulier celles de Iglicka (1999), Sword (1999), Sik et Wallace (1999), Czakó et Sik (1999), Wallace et alii (1999). Alors que le petit commerce entre la Pologne et l'Allemagne, apanage des Polonais, ralentissait avec le renforcement du zloty polonais et de l'ajustement progressif des prix des deux côtés de la frontière, le commerce à la frontière Est a gagné en importance. Sword (1999 151) notamment a montré que la valeur des biens achetés par les petits revendeurs en Pologne était égale à $46 \%$ de celle des exportations officielles vers l'Ukraine et à $159 \%$ de celles vers la Biélorussie en 1996. Les réseaux de commerce passèrent progressivement des mains des ressortissants de l'Europe centrale aux mains des citoyens de la CEI et de certains ressortissants asiatiques (Vietnamiens et Chinois en particulier). Le petit commerce international enfreignant les réglementations fiscales et douanières des pays de destination et d'origine, la plupart des revendeurs se sont « déguisés en touristes » pour passer la frontière. Ainsi Okolski (1998) a estimé que la " grande majorité » des 500000 Ukrainiens qui se sont rendus en Pologne étaient des revendeurs.

Parmi les acteurs de ce commerce transfrontalier, deux catégories doivent être distinguées. Les plus puissants, les gatekeepers (intermédiaires) venaient de la bureaucratie d'État et exploitaient leur capital social. Ils nouaient des contacts et contrôlaient la plupart des échanges via un système de faux permis et l'instauration de règlements particuliers. Parfois ils jouaient aussi le rôle d'entrepreneurs (voyagistes aux activités plus ou moins licites), facilitant ainsi le travail des opérateurs. Ceux-ci étaient des commerçants à "la petite semaine", qui faisaient de courts voyages à l'étranger puis revendaient pour leur propre compte les produits achetés sur les marchés locaux. La plupart des marchandises étaient « de qualité médiocre et d'origine 
douteuse » (Czakó et Sik, 1999 718). Notre enquête a porté sur les deux groupes, mais dans cet article nous ne traitons que des opérateurs (petits commerçants), afin d'établir une comparaison avec les autres formes de mobilité internationale. Les conclusions de Czakó et Sik (1999), Sik et Wallace (1999), Wallace et alii (1999), Sword (1999), Iglicka (1999) et de nos propres travaux sur les travailleurs migrants ukrainiens et les commerçants mettaient en évidence de nombreuses similitudes entre le petit commerce transfrontalier et la migration de travail à l'étranger. Nous avons voulu tester deux hypothèses

29 - Les origines sociales et économiques des commerçants et des travailleurs migrants étaient-elles similaires?

30 - Si oui, le commerce répondait-il aux mêmes motivations que la migration de maind'œuvre?

31 - Les comportements d'épargne et de dépense étaient-ils similaires dans les deux échantillons?

Champ d'étude et méthodologie

32 Notre étude, conduite à l'automne 1999 sur des marchés locaux dans les villes de Uzhgorod et Irschava, en Ukraine occidentale, comportait un échantillon de 150 commerçants. La ville ukrainienne de Uzhgorod s'étend à la frontière entre la Slovaquie et l'Ukraine. Elle est un lieu de passage important pour la main-d'œuvre migrante, les petits commerçants et les touristes. Les marchandises vendues sur les marchés avaient été achetées majoritairement dans les pays voisins (Hongrie, Slovaquie et Pologne), mais certains commerçants se rendaient aussi jusqu'en Europe de l'ouest ou en Turquie pour se ravitailler. L'enquête sur les commerçants a été menée avec les mêmes méthodes que celle sur les migrants.

Résultats de l'enquête

33 Les commerçants ukrainiens présentaient des profils similaires à ceux des travailleurs migrants de l'enquête précédente. Ils étaient jeunes (62\% âgés de moins de 35 ans) et les hommes représentaient 59 \% de l'échantillon. La structure par sexe et par âge était identique à celle trouvée par Czakó et Sik (1999 727) dans leur étude sur les commerçants ukrainiens en Hongrie. La recherche menée par Iglicka (1999) sur les commerçants ukrainiens en Pologne a trouvé les mêmes résultats concernant la structure par âge mais des pourcentages différents de femmes. Des similitudes existaient avec notre propre étude sur les migrants ukrainiens en Slovaquie $39 \%$ des commerçants déclaraient un niveau d'études supérieures et $29 \%$ un niveau secondaire. Comme dans l'enquête sur les travailleurs migrants, le niveau d'études n'était corrélé ni au sexe, ni au type d'emploi, ni au revenu; il semble que la situation économique de l'Ukraine ait effacé la plupart des distinctions fondées sur le niveau d'études. Alors que les commerçants, comme les travailleurs, étaient plutôt instruits, ils n'avaient que peu d'occasions d'utiliser leurs connaissances dans le type d'activité qu'ils exerçaient. Quant au dernier emploi occupé en Ukraine, les commerçants présentaient des profils divers enseignants et conférenciers (12\%), ingénieurs diplômés (4\%), étudiants (5\%) et ouvriers (22\%) représentaient les professions les plus courantes, mais l'échantillon comprenait également un médecin, un cadre, un expert comptable, une femme au foyer et un journaliste. Les femmes étaient légèrement plus jeunes que les hommes, mais avaient un niveau d'études plus élevé plus de la moitié d'entre elles possédait un diplôme universitaire (tableau 4). 
Tableau 4 : Petits commerçants transfrontaliers ukrainiens. Coefficients de corrélation

\begin{tabular}{|l|c|c|c|c|c|c|c|c|}
\hline $\begin{array}{l}\text { Petits } \\
\text { commerçants } \\
\text { ukrainiens }\end{array}$ & Sexe & Âge & Études & Principale & Méthode & Conditions & Raison & Avenir \\
\hline Sexe & $\mathrm{x}$ & $\mathrm{x}$ & $\mathrm{x}$ & $\mathrm{x}$ & $\mathrm{x}$ & $\mathrm{x}$ & $\mathrm{x}$ & $\mathrm{x}$ \\
\hline Âge & 0.170 & $\mathrm{x}$ & $\mathrm{x}$ & $\mathrm{x}$ & $\mathrm{x}$ & $\mathrm{x}$ & $\mathrm{x}$ & $\mathrm{x}$ \\
\hline Études & 0.176 & $\mathrm{n}$ & $\mathrm{x}$ & $\mathrm{x}$ & $\mathrm{x}$ & $\mathrm{x}$ & $\mathrm{x}$ & $\mathrm{x}$ \\
\hline Principale & $\mathrm{n}$ & $\mathrm{n}$ & $\mathrm{n}$ & $\mathrm{x}$ & $\mathrm{x}$ & $\mathrm{x}$ & $\mathrm{x}$ & $\mathrm{x}$ \\
\hline Méthode & 0.288 & $\mathrm{n}$ & $\mathrm{n}$ & $\mathrm{n}$ & $\mathrm{x}$ & $\mathrm{x}$ & $\mathrm{x}$ & $\mathrm{x}$ \\
\hline Conditions & $\mathrm{n}$ & $\mathrm{n}$ & $\mathrm{n}$ & 0.170 & $\mathrm{n}$ & $\mathrm{x}$ & $\mathrm{x}$ & $\mathrm{x}$ \\
\hline Raison & 0.250 & $\mathrm{n}$ & $\mathrm{n}$ & $\mathrm{n}$ & $\mathrm{n}$ & $\mathrm{n}$ & $\mathrm{x}$ & $\mathrm{x}$ \\
\hline Avenir & $\mathrm{n}$ & $\mathrm{n}$ & $\mathrm{n}$ & 0.218 & $\mathrm{n}$ & $\mathrm{n}$ & $\mathrm{n}$ & $\mathrm{x}$ \\
\hline
\end{tabular}

Réponses uniques. Seules les corrélations ayant un niveau de signification à 0.05 ont été reportées. $\mathrm{n}=$ non significatif.

Sexe : $1:$ homme, $2:$ femme ; Âge : $1:$ jusqu'à 34 ans, $2: 35$ et plus ; Etudes : $1:$ primaire, secondaire $1^{\text {er }}$ et $2^{\mathrm{em}}$ cycle, 2 : université ; Principale (commerce comme source principale de revenus) : $1:$ oui, $2:$ non ; méthode (de travail) : $1:$ voyages en bus, $2:$ voiture personnelle ou paiement d'autres personnes ; Conditions (de travail) : 1 : se sont améliorées ou sont restées les mêmes, 2 : ont empire ; Raison (pour lesquelles les conditions ont empiré) : 1 : baisse du pouvoir d'achat de la population ; 2 : trop de formalités administratives, frais de douane ou concurrents ; Futur (projets de vie) : 'Je vais exercer ce travail ou je vais changer de domaine', 2 : 'Je vais vivre dans un autre pays'.

Source : enquêtes des auteurs, 1999

Les commerçants avaient trois façons principales d'organiser l'achat de leurs marchandises $30 \%$ d'entre eux se déplaçaient en utilisant les transports publics ou à des cars de tourisme (forme la plus élémentaire d'organisation du commerce), tandis que $45 \%$ se servaient de leur propre voiture et $25 \%$ payaient d'autres personnes pour se déplacer à leur place. La fréquence de chacun de ces trois modes d'organisation n'était pas la même pour les femmes, dont $48 \%$ utilisaient les transports en commun, que pour les hommes chez qui cette proportion n'était que de $19 \%$.

Le commerce était la source principale des revenus de la plupart (71\%) des personnes que nous avons interrogées. La faiblesse du statut attribué à cette activité et le niveau réduit des bénéfices expliquent probablement des réticences des répondants ils étaient beaucoup moins disposés à nous parler de leurs revenus que les travailleurs migrants. Néanmoins nous avons recueilli des réponses aux questions concernant ceux-ci. Peu d'entre eux ont rendu compte d'un accroissement significatif de leurs revenus depuis le début de leur activité. Seuls $12 \%$ ont déclaré avoir acheté une maison ou un appartement. Un plus grand nombre de commerçants $(23 \%)$ a déclaré avoir acheté une voiture, proportion proche de celle des commerçants utilisant leur propre voiture pour leur activité. Ceci ne signifie pas que le petit commerce international n'était pas une activité rentable, mais la plus grande part des profits était retenue par les gatekeepers, qui, eux, se sont faits construire de luxueuses maisons dans les faubourgs de Uzhgorod.

Au moment de l'enquête, les commerçants à «la petite semaine » se sont retrouvés dans une situation difficile. $85 \%$ d'entre eux déclaraient que les conditions de travail avaient empiré au cours des trois dernières années, alors que seuls $3 \%$ pensaient s'en sortir mieux. La baisse du pouvoir d'achat de la clientèle potentielle était considérée comme la raison principale de la dégradation des conditions de travail (71\%), suivie de l'augmentation du nombre de réglementations administratives des deux côtés de la frontière (12\%), l'augmentation du nombre de concurrents, et les fortes taxes imposées par l'État ukrainien. La baisse du pouvoir d'achat était essentiellement ressentie par les 
femmes commerçantes et migrantes qui opéraient à l'extérieur de l'Europe de l'ouest. Malgré ces conditions de travail, près de la moitié des commerçants ( $40 \%)$ voulaient persévérer dans cette activité mais $36 \%$ souhaitaient au contraire rechercher un autre emploi en Ukraine. Les personnes, qui déclaraient le commerce comme leur source principale de revenus, avaient tendance à être plus optimistes et voulaient continuer ce travail. En outre, $24 \%$ des commerçants interrogés étaient désireux de s'établir à l'étranger, et ce groupe coïncidait avec celui des personnes qui n'accumulait aucune richesse. Les projets d'émigration étaient plutôt le fait de personnes jeunes. Des témoignages similaires se trouvent dans l'étude effectuée par Iglicka (1999 136) sur les ressortissants de la CEI en Pologne ${ }^{2}$. Ces faits suggèrent que le petit commerce est davantage une stratégie de survie qu'une source de revenus durable.

Des entretiens avec des opérateurs et des gatekeepers ont révélé des transformations dans les activités d'une partie des commerçants transfrontaliers. Certains opérateurs qui avaient accumulé du capital ont remplacé les voyages fréquents destinés à ramener de petites quantités de marchandises par l'organisation d'opérations d'importation à grande échelle à partir du Sud-Est asiatique et de la Turquie. Les cargaisons étaient livrées par bateau dans les ports du Sud de l'Ukraine et/ou ou par camion dans d'autres villes ukrainiennes. D'autres commerçants se spécialisaient dans le re-conditionnement de grosses cargaisons en quantités plus petites et les réexportaient vers les économies de transition d'Europe centrale et des États baltes.

Cependant, la plupart des opérateurs manquaient de ressources financières et/ou de compétence et perpétuaient une forme classique de petit commerce. Il serait peu réaliste de s'attendre à une fin rapide du petit commerce transfrontalier et à son remplacement par des réseaux conventionnels de vente au détail. Dans des pays où les salaires étaient payés avec six mois de retard, voire n'étaient jamais versés, le commerce offrait une source alternative de revenus, qui ne demandait ni investissement important de capital, ni connaissances élaborées. Wallace (1998), par exemple, a estimé que seulement $10 \%$ de la population ukrainienne étaient en mesure de vivre avec les seuls revenus de leur emploi principal. La situation a peu changé au cours des premières années $d u x x^{e}$ siècle la récession économique persistante et l'agitation politique ont laissé peu de chances à l'Ukraine de rattraper ses voisins plus heureux d'Europe centrale. Tant que la frontière entre l'Europe centrale et l'Europe de l'est reste une frontière entre deux systèmes socio-économiques contrastés, le commerce reste une possibilité de survie modeste mais bienvenue pour de nombreuses familles.

Tourisme et achats les slovaques en Autriche et en Hongrie

Le tourisme inclut une large palette d'activités, parmi lesquelles les loisirs, les affaires et le "shopping ", les achats sont les plus importantes. Pour les voyageurs issus des pays européens développés, les loisirs ou les affaires expliquent l'essentiel des déplacements (Williams et Shaw 1998). En Europe centrale et de l'Est, communiste et postcommuniste, les raisons de voyager ont été assez différentes.

À l'exception du tourisme classique (loisirs, intérêts personnels, etc.), la population des pays communistes utilisait les voyages internationaux comme un moyen de pallier les faiblesses des organisations de commerce d'État dans l'approvisionnement en biens de consommation. On rappellera que les touristes tchèques et slovaques s'approvisionnaient dans les magasins hongrois à l'époque du "socialisme goulasch ", et que les commerçants polonais achetaient des produits occidentaux en Allemagne et 
en Autriche pour les revendre en Pologne. Ces pratiques était d'ailleurs très prisées par les membres de l'armée russe dans les pays membres du Pacte de Varsovie (Jerczynski, 1999). Ce type de tourisme était très important, et même officiellement encouragé en Europe centrale et de l'Est.

41 Avec la suppression des visas et du passeport au début des années quatre-vingt-dix, les ressortissants de l'Europe centrale ont vécu une courte période d'augmentation du volume de petit commerce. Les frontières entre la Pologne et l'Allemagne et entre la Tchéquie et l'Allemagne ont été au centre de ce commerce «informel». La fin de la récession économique et la hausse du niveau de vie en Europe centrale dès le milieu de ces années quatre-vingt-dix a amené d'importants changements sociaux et géographiques dans les flux de population dans la région. Les ressortissants de l'Europe centrale ont abandonné le " commerce à la valise » et se sont intéressés de plus en plus aux formes classiques du voyage de loisirs dans les pays d'Europe de l'ouest en général et méditerranéens en particulier. Le "commerce à la valise" n'a pas diminué pour autant ; il s'est déplacé vers les frontières ukrainiennes. Par contre, les ressortissants de l'Europe centrale ont continué à pratiquer le "tourisme shopping», le tourisme d'achats, destiné à leur consommation personnelle (Böröcz, 1996; Williams et Balá 2001). Cette activité représentait une part importante de l'ensemble des voyages touristiques et des dépenses que ceux-ci engendraient.

42 On peut se demander pourquoi la part de ce type de tourisme dans le commerce international total était si élevé ? Plusieurs réponses sont possibles

43 - la faiblesse des réseaux des grandes chaînes internationales de vente au détail qui se sont établis dans la région,

44 - le peu de capital nécessaire et les faibles coûts de transaction des achats courants,

45 - les différences de prix entre les différents pays concernés,

46 - le bas niveau de vie dans certaines régions frontalières.

47 Nous avons exploré l'importance du «tourisme d'achats» aux frontières slovaques avec l'Autriche et la Hongrie lors de deux enquêtes. Les rapports réalisés par l'Institut du Tourisme (Ústav turizmu 1997) et le Ministère de l'Économie slovaque, Section Tourisme (MHSR 2000) nous ont fourni des informations intéressantes sur les entrées et les sorties en Slovaquie, selon la nationalité et le motif du déplacement. Nous avons pu vérifier ces données durant notre travail de terrain en Slovaquie à l'automne 1999. Après des entretiens avec les autorités responsables des contrôles nous avons élaboré un questionnaire destiné aux voyageurs. Différentes hypothèses ont été mises à l'épreuve

48 - quel type de tourisme se pratiquait-il à chacune de ces deux frontières? La nature de la frontière joue-t-elle un rôle?

49 - nous connaissions l'importance du "tourisme d'achats» durant la période communiste. Cette pratique perdurait-elles?

50 - y avait-il un ou plusieurs types de «tourisme d'achats » dans un même pays ? S'il y en avait plusieurs, quelles sont les populations et les régions qui les pratiquaient?

Champ d'étude et méthodologie

51 La première enquête a eu lieu à l'automne 1999 sur un échantillon de 108 voyageurs, aux points de passage frontaliers de Petralka-Berg et de Jarovce-Kittsee entre la Slovaquie et l'Autriche. La seconde enquête a été menée durant la même période aux 
points de passage frontaliers de Bratislava-Rusovce et de Komárno-Komárom entre la Slovaquie et la Hongrie, sur un échantillon de 121 voyageurs. Ces points de passage enregistraient les pourcentages les plus élevés de mobilité entre la Slovaquie et la Hongrie. Avec l'aide du Service des Garde-Frontières, les voitures slovaques se rendant en Autriche, ont été arrêtées et les conducteurs interrogés sur la raison de leur voyage. Si celle-ci n'était pas liée au travail, des questions sur la fréquence des déplacements et les dépenses touristiques ont été posées. L'enquête a été traitée selon les mêmes méthodes statistiques que celle sur la migration de travail vers l'Autriche.

Résultats de l'enquête

En considérant les deux échantillons, on observe que la plupart des voyageurs étaient des hommes ( $79 \%$ à la frontière autrichienne et $60 \%$ à la frontière hongroise). La moitié des voyageurs avait moins de 35 ans ( 48 et $47 \%$ selon la frontière), la tranche d'âge 25-34 ans étant la plus fréquente. La structure par âge expliquait partiellement le statut familial environ les deux tiers des voyageurs étaient mariés. Cependant, les raisons et la fréquence des déplacements, ainsi que les dépenses par individu, différaient de façon significative.

La plus grande partie de ceux qui se rendaient en Autriche déclarait les achats $(38 \%)$ et les affaires $(29 \%)$ comme principaux motifs de leur déplacement. Les autres raisons déclarées étaient le transit (17\%), le tourisme de loisir (12\%), et les études (4\%). La part du tourisme de loisir peut sembler faible, mais la période de l'année et le prix relativement élevé du tourisme en Autriche peuvent l'expliquer. Le nombre de voyageurs pour affaires ou en transit, dont la plupart rejoignait l'aéroport de Vienne, expliquait le pourcentage relativement élevé de diplômés (baccalauréat $57 \%$, diplôme universitaire $22 \%$ ). Il y avait une forte corrélation entre les motifs du déplacement et les dépenses effectuées en Autriche. Les touristes se rendant en Autriche pour effectuer des achats ou pour les loisirs dépensaient beaucoup plus que les autres (tableau 5). Pour les voyageurs vers la Hongrie, les achats constituaient le motif principal du déplacement à un degré bien plus élevé que dans le cas de l'Autriche (63\%). Le tourisme de loisirs (19\%), la visite de membres de la famille ou d'amis (9\%), les affaires (7\%) et le transit $(2 \%)$ étaient moins fréquents aux frontières hongroises. Les voyageurs en transit, pour affaires ou rendant visite à leur famille ou à des amis avaient tendance à être plus vieux que ceux qui se déplaçaient pour effectuer des achats et pratiquer le tourisme de loisir.

La fréquence des déplacement était élevée dans les deux enquêtes $18 \%$ de ceux qui se rendaient en Autriche allaient faire des achats au moins une fois par semaine, et $30 \%$ au moins une fois par mois. Les voyageurs qui allaient en Hongrie déclaraient des fréquences similaires (respectivement 16 et $26 \%$ ). En outre les habitants de Bratislava voyageait plus fréquemment que ceux des autres régions.

Alors que nous attendions une distribution relativement proportionnelle du trafic entre les différentes régions slovaques, les voyageurs venant de Slovaquie occidentale étaient largement sur-représentés dans l'échantillon autrichien. Plus de la moitié d'entre eux venaient de Bratislava, (ville $43 \%$, reste de la région $13 \%$ ) ; $8 \%$ de chacune des régions de Trenín et Trnava. Enfin seuls 7 et $6 \%$ des voyageurs venaient des régions de Dunajská Streda et Galanta (proches de la frontière hongroise et dans lesquelles la minorité ethnique hongroise est bien représentée (figure 1). Au total, $85 \%$ des voyageurs venaient de ces six régions. Il n'y avait que quelques voitures immatriculées dans d'autres villes et régions industrielles slovaques (Košice, Prešov, Banská Bystrica, 
etc.). Ce n'est pas la situation du poste frontière qui expliquait cette distribution tous les voyageurs vers l'Autriche devaient emprunter le point de passage frontalier de Bratislava. Ce sont plutôt les contrastes de la géographie économique slovaque qui le justifiaient ainsi l'autoroute slovaque de Bratislava-Nord, ainsi que la plupart des activités commerciales, ne se prolongeaient pas au delà de la région de Trenín.

Une concentration régionale similaire a été trouvée pour les voyageurs à la frontière slovaquo-hongroise. La plupart des déplacements transfrontaliers avaient pour origine les régions frontalières, adjacentes aux points de passage concernés. $84 \%$ des voyageurs venaient de quatre régions Bratislava-ville (40\%), sa région (8), Komárno (26) et Nové Zámky (11) (figure).

57 Le niveau de vie à Bratislava étant bien meilleur que celui des régions de la frontière hongroise, il n'est pas étonnant de constater que, dans les deux échantillons, les habitants de la capitale dépensaient plus d'argent par voyage que ceux des autres villes. D'autre part, les prix étant plus élevés en Autriche qu'en Hongrie, on pouvait penser que les dépenses par voyage obéiraient à la même hiérarchie, mais c'est le contraire qui s'est vérifié la dépense moyenne par voyage était plus élevée en Hongrie.

Les voyages vers la Hongrie avaient pour principal but d'effectuer des achats. Les produits les plus recherchés par les voyageurs slovaques étaient d'abord les vêtements, les articles en cuir et les chaussures, qui ensemble représentaient $41 \%$ des dépenses. On trouvait ensuite la nourriture $(25 \%)$, d'autres marchandises et des boissons alcoolisées. Il était intéressant de constater que les femmes dépensaient généralement beaucoup plus que les hommes, ce qui était probablement lié au rôle traditionnel de la femme comme " ministre des finances » dans les familles. Les vêtements, les articles en cuir et les chaussures étaient essentiellement achetés par les générations plus jeunes, le plus souvent lors de voyages d'une journée et par des personnes effectuant de courtes distances. Ces biens étaient de 25 à $50 \%$ moins chers en Hongrie qu'en Slovaquie. Ces résultats donnent l'image d'une famille jeune effectuant un court voyage de l'autre côté de la frontière pour y acheter des vêtements. 
Figure 1 Origine des voyageurs slovaques (par district) aux frontières de l'Autriche et de la Hongrie en 1999

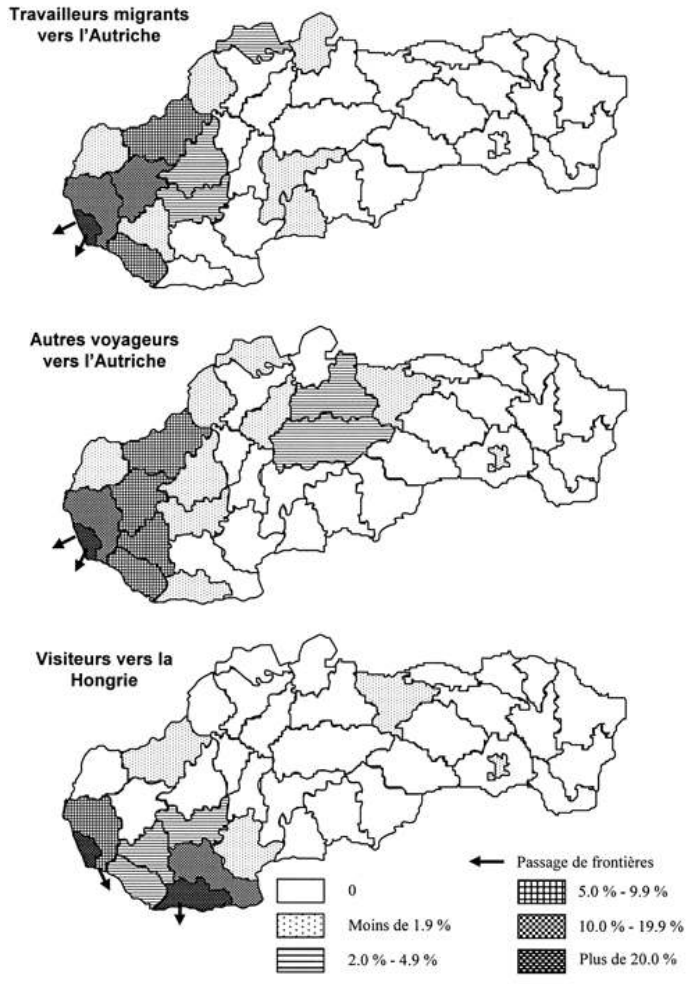

Source enquête des auteurs, 1999

Tableau 5 : Dépenses des voyageurs slovaques pour leurs achats en Autriche et en Hongrie

\begin{tabular}{|l|c|c|}
\hline Dépenses (en US\$) & Autriche & Hongrie \\
\hline Moins de 25 & 40,7 & 31,6 \\
\hline 25 à 124 & 39,8 & 52,0 \\
\hline 125 à 249 & 16,7 & 11,1 \\
\hline 250 et plus & 2,8 & 5,1 \\
\hline
\end{tabular}

Source : enquêtes des auteurs, 1999

Nous pouvons distinguer ainsi deux types différents de "tourisme d'achats » à l'intérieur du même pays. Les magasins de Vienne étaient essentiellement visités par des personnes à hauts revenus (venus principalement de Bratislava), qui recherchaient un choix plus varié et une meilleure qualité dans les marchandises. Paradoxalement, ce type de tourisme était un héritage de la période communiste. Le second type était le fait de familles aux revenus peu élevés, venant des régions pauvres, et qui cherchaient en Hongrie des magasins bon marché, notamment des marchés en plein air. Ce type de tourisme d'achats était lié aux déséquilibres régionaux générés par la transition vers une économie de marché. Alors que la redistribution des bénéfices avait fondé la politique régionale durant la période communiste, c'est la compétition entre régions face aux possibilités d'investissement et d'emploi qui l'emporte après 1989. En Slovaquie, Bratislava a été la principale bénéficiaire de cette nouvelle politique, tandis que les autres régions à la frontière sud étaient les perdantes. Le PIB par habitant était de 8000 \$US à Bratislava, mais seulement de 1700 et 3000 \$US dans les régions de Komárno et Nové Zámky en 1996 (dernières données disponibles, PIB calculé d'après les 
taux de change). La comparaison des taux de chômage à Bratislava-ville et dans les régions de Komárno (23.8\%) et Nové Zámky (23.7\%) suggérait également des différences de compositions sociales et des motifs de déplacement pour achats différents selon les régions.

Notre enquête a révélé une forte spécificité régionale dans les déplacements transfrontaliers aux frontières Slovaquie-Autriche et Slovaquie-Hongrie. Les motifs et les habitudes de voyage dans la région étaient fortement déterminés par les capacités culturelles et économiques, et aussi les « habitus » ethniques.

61 À la frontière entre la Slovaquie et la Pologne, la situation est proche de celle constatée à la frontière slovaquo-hongroise. La plupart des trois millions de voyageurs slovaques qui traversent chaque année la frontière étaient intéressés par l'acquisition de biens de consommation. Cependant, les articles achetés dans les bazars polonais n'étaient pas produits en Pologne, mais venaient pour la plupart d'autres parties du monde (principalement de l'Asie). Ils étaient également disponibles en Slovaquie, mais plus chers car taxes et droits de douane y étaient plus élevés.

Les chaînes internationales de vente au détail ont joué aussi un rôle important dans le développement du commerce transfrontalier des chaînes britanniques, allemandes, autrichiennes et françaises (TESCO, Carrefour, BILLA) ont établi des filiales en Hongrie, en Pologne et dans la République Tchèque au milieu des années quatre-vingt-dix, tandis que la Slovaquie restait loin derrière. Les déplacements d'une journée vers les centres commerciaux hongrois et tchèques ont pris alors de l'ampleur pour de nombreuses familles slovaques.

Tableau 6 : Voyageurs slovaques en Autriche et en Hongrie. Coefficients de corrélation

\begin{tabular}{|l|c|c|c|c|c|c|}
\hline $\begin{array}{l}\text { Voyageurs } \\
\text { slovaques en Autriche }\end{array}$ & Sexe & Âge & Motif & Fréquence & Dépenses & Bratislava \\
\hline Sexe & $\mathrm{x}$ & $\mathrm{x}$ & $\mathrm{x}$ & $\mathrm{x}$ & $\mathrm{x}$ & $\mathrm{x}$ \\
\hline Âge & $\mathrm{n}$ & $\mathrm{x}$ & $\mathrm{x}$ & $\mathrm{x}$ & $\mathrm{x}$ & $\mathrm{x}$ \\
\hline Motif & $\mathrm{n}$ & $\mathrm{n}$ & $\mathrm{x}$ & $\mathrm{x}$ & $\mathrm{x}$ & $\mathrm{x}$ \\
\hline Fréquence & $\mathrm{n}$ & $\mathrm{n}$ & $\mathrm{n}$ & $\mathrm{x}$ & $\mathrm{x}$ & $\mathrm{x}$ \\
\hline Dépenses & $\mathrm{n}$ & $\mathrm{n}$ & 0.427 & $\mathrm{n}$ & $\mathrm{x}$ & $\mathrm{x}$ \\
\hline Bratislava & $\mathrm{n}$ & $\mathrm{n}$ & $\mathrm{n}$ & 0.206 & 0.186 & $\mathrm{x}$ \\
\hline $\begin{array}{l}\text { Voyageurs } \\
\text { slovaques en Hongrie }\end{array}$ & Sexe & $\mathrm{Age}$ & Motif & Fréquence & Dépenses & Bratislava \\
\hline Sexe & $\mathrm{x}$ & $\mathrm{x}$ & $\mathrm{x}$ & $\mathrm{x}$ & $\mathrm{x}$ & $\mathrm{x}$ \\
\hline Âge & $\mathrm{n}$ & $\mathrm{x}$ & $\mathrm{x}$ & $\mathrm{x}$ & $\mathrm{x}$ & $\mathrm{x}$ \\
\hline Motif & $\mathrm{n}$ & 0.247 & $\mathrm{x}$ & $\mathrm{x}$ & $\mathrm{x}$ & $\mathrm{x}$ \\
\hline Fréquence & $\mathrm{n}$ & $\mathrm{n}$ & $\mathrm{n}$ & $\mathrm{x}$ & $\mathrm{x}$ & $\mathrm{x}$ \\
\hline Dépenses & $\mathrm{n}$ & $\mathrm{n}$ & $\mathrm{n}$ & 0.337 & $\mathrm{n}$ & $\mathrm{x}$ \\
\hline Bratislava & $\mathrm{n}$ & $\mathrm{n}$ & $\mathrm{n}$ & $\mathrm{x}$ & $\mathrm{x}$ \\
\hline
\end{tabular}

Réponses uniques. Seules les corrélations ayant un niveau de signification à 0.05 ont été reportées. $\mathrm{n}=$ non significatif.

Sexe : $1:$ homme, $2:$ femme ; Âge : $1:$ jusqu'à 34 ans, $2: 35$ et plus ; Motif (de voyage) : 1 : transit, affaires et études, 2 : achats et tourisme ; Fréquence (de voyage en Autriche ou en Hongrie) : 1 : au moins une fois par mois, $2:$ moins souvent ; Dépenses : (par tête et par voyage) 1 : jusqu'à $1000 \mathrm{Sk}$ (25 \$US), 2 : plus de $1000 \mathrm{Sk}$; Bratislava : 1: voiture originaire de Bratislava-ville, 2 : autres voitures.

Source : enquêtes des auteurs, 1999

63 L'importance du tourisme d'achats transfrontalier a été reconnue par les autorités slovaques le Programme National de Développement Touristique (MHSR 2000) a estimé 
de manière crédible que les revenus et les dépenses du tourisme international, tels qu'ils étaient définis par les méthodes de l'enquête, étaient trois fois plus importants que les résultats enregistrés par la Banque Nationale Slovaque. Nos enquêtes ont permis d'estimer des rapports similaires entre bilan officiel du tourisme et bilan nonofficiel, et les rapports restent proches pour les enquêtes réalisées en Tchèquie et en Hongrie (pour plus de détails, voir Williams et Balá, 2000). Le Programme National de Développement a estimé le solde négatif du " tourisme d'achats » international à 13,7 milliards Sk (400 millions \$US) en 1997 pour la Slovaquie. Le solde promettait d'être supérieur à la fin de la décennie, étant donné la vitesse de construction de centres commerciaux internationaux, devenus la cible principale des déplacements pour les citoyens slovaques.

Des sociétés différentes génèrent des modèles de mobilité différents

L'ouverture des frontières entre l'Europe centrale et l'Europe de l'ouest, au début des années quatre-vingt-dix s'est accompagnée de prédictions alarmistes sur une émigration massive des pays post-communistes vers la zone de l'Union Européenne. Ces prédictions se sont révélées fausses. Une analyse quantitative des mouvements de population entre l'Europe centrale et l'Europe de l'ouest a montré que si la mobilité globale (exprimée en nombre de passages de frontières) avait augmenté, l'accroissement des migrations permanentes était beaucoup moins spectaculaire. $\mathrm{Ce}$ sont les déplacements temporaires des ressortissants des pays en transition qui représentent, et de loin, le type le plus important de mobilité transfrontalière. Ils incluent à la fois la migration temporaire de main-d'œuvre et les déplacements transfrontaliers de courte durée. La majorité des déplacements sont motivés par le tourisme (y compris pour achats), suivi par le petit commerce et le travail temporaire, ponctuel ou saisonnier, à l'étranger. Les motivations de ces courants varient d'un pays à l'autre les populations les plus riches (Tchèques, Slovaques, Polonais et Hongrois) enregistrent des pourcentages très élevés de voyages touristiques et de déplacements pour acheter des marchandises, tandis que les ressortissants des pays plus pauvres (Ukrainiens et autres ressortissants de la CEI) se déplacent à l'étranger pour faire du commerce ou pour participer à des activités illégales.

Une mobilité intense mais pas d'émigration massive

Selon les théories néo-classiques, on pouvait s'attendre à ce que de grandes différences dans les revenus génèrent des flux élevés de migration permanente. Les chiffres réels et la distribution des migrants ne suivent ni les scénarios catastrophiques ni les prévisions fondées sur l'ouverture des frontières entre l'Europe centrale et l'Europe de l'ouest. Soixante millions de ressortissants de l'Europe centrale ont traversé les frontières de l'Union Européenne en 2000, mais la main-d'œuvre migrante ne constitue qu'une petite fraction de ces déplacements. Les salaires mensuels en Europe centrale, autour de 300 \$US peuvent sembler bas lorsqu'on les compare à ceux de l'Europe de l'ouest, mais ils permettent un niveau de vie décent car les prix locaux sont peu élevés. Le dossier publié dans le rapport SOPEMI de 1998 a estimé qu'en 1996, 55000 Tchèques et Slovaques, 80000 Hongrois et 420000 Polonais vivaient légalement dans la zone de l'Union Européenne et en Suisse, effectifs réduits si on les compare aux 2.1 millions d'ex-Yougoslaves et aux millions de Turcs, Kurdes et ressortissants d'autres pays asiatiques. Il est clair que les principaux flux migratoires vers les pays de l'Ouest sont d'abord liés aux conflits, guerres civiles et persécutions politiques. D'autre part, l'Europe centrale est devenue à la fois une région de destination et de transit avec un solde migratoire positif. On compte par exemple en République Tchèque 140000 
étrangers ayant un permis de travail, auquel il faut ajouter entre 50000 et 70000 travailleurs en situation irrégulière (le plus souvent Ukrainiens) y demeurant illégalement (OECD 1998).

Plusieurs facteurs se sont combinés pour aboutir à cette situation, à peine envisagée en 1989. Les pays post-communistes d'Europe centrale ont réussi, malgré les difficultés de la première période de transition, à préserver une stabilité politique et sociale et à maintenir un niveau de vie acceptable. Cette situation a dissuadé les populations d'émigrer. En même temps, ces pays ont attiré des habitants pauvres de l'ancienne Union Soviétique. Wallace et alii (1999 754) ont tenté de démontrer que les économies de transition de l'Europe centrale et des États baltes étaient « de plus en plus dans l'orbite économique de l'Europe de l'ouest, tandis que celles de l'Est s'enfonçaient de plus en plus dans une situation économique désorganisée où les régulations informelles, y compris le capital social, sont devenues un principe dominant d'organisation ». De plus, l'Union Européenne et les autres pays d'Europe de l'ouest ont appliqué aux populations des pays postcommunistes de l'Europe centrale des politiques migratoires raisonnables. Leurs ressortissants ont obtenu facilement des autorisations de séjour de courte durée (moins de trois mois), ce qui a facilité le développement des voyages pour affaires et du tourisme, et favorisé l'acquisition de biens. Parfois, les voyages touristiques ont été l'occasion d'une recherche illégale d'emploi, mais le plus souvent pour des périodes limitées. Ces politiques ont contribué à la prévention d'une potentielle immigration de main-d'œuvre à grande échelle et à sur le long terme.

Les transformations du marché du travail dans l'Union Européenne comme en Europe centrale ont joué un rôle important dans l'installation de cette situation migratoire imprévue. L'Union Européenne exprimait une demande croissante de main-d'œuvre qualifiée et instruite, et les enquêtes sur les migrants tchèques, slovaques et polonais ont confirmé les pourcentages élevés de migrants possédant un niveau d'études supérieures. Beaucoup de migrants se comportaient selon les modèles suggérés par la théorie du marché dual et acceptaient des emplois peu qualifiés en émigration. Cependant, un certain nombre de migrants instruits était intéressés par des emplois hautement qualifiés et rémunérés. Il était également possible de discerner un passage de la migration permanente ou à long terme à des emplois de courte durée. Il est intéressant de noter que la préférence pour un mode de vie, que l'on peut résumer par la formule, "travailler là-bas, vivre ici ", était exprimée à la fois par les migrants occupant des emplois qualifiés et non-qualifiés, travailleurs saisonniers polonais en Allemagne (Korczynska 1997b) ou Slovaques rencontrés lors de nos enquêtes.

Dans la migration de l'Europe centrale vers l'Union Européenne comme dans celle de l'Europe de l'est vers l'Europe centrale, la fuite des cerveaux représente une composante importante. On peut même parler de "gaspillage » des cerveaux dans le cas de la migration de ressortissants de la CEI vers la Pologne (Domaradzka, 1997) et de Polonais vers l'Allemagne (Korczynska, 1997a et 1997b) ou la Hollande (Odé et Knaap, 1998 215-7). La situation peut être meilleure lorsqu'il existe à la fois des similitudes et une proximité géographique entre les régions d'origine et de destination de la maind'œuvre. Ainsi la distance entre Bratislava et Vienne, deux grands centres urbains de production, d'études et de recherche, permet une navette quotidienne de la maind'œuvre qualifiée et instruite les niveaux de déqualification sont relativement bas.

Deux modèles de mobilité 

l'Europe de l'ouest l'amélioration de leur statut social. La plus grande partie de l'argent gagné à l'étranger est destinée à l'achat ou à la construction d'une nouvelle maison, à l'acquisition d'une voiture, à l'épargne. Les revenus tirés de leur travail, plus élevés que ceux des migrants de la CEI, leur permettent ces investissements. Il est vrai qu'il y a des différences entre les migrants et les commerçants, ces derniers étant plus pauvres et davantage contraints à la déqualification. Parmi les commerçants il y a une proportion relativement élevée de jeunes femmes instruites qui, malgré son faible rendement, ont choisi cette activité car elle demande moins de temps.

列 l'Europe centrale travaillant à l'étranger. L'une d'elles était la part relativement faible de personnes désireuses de quitter leur pays pour toujours la plupart des migrants et des commerçants voient leur occupation actuelle comme temporaire et voulaient retourner dans leur lieu d'origine. Les migrants et les commerçants ukrainiens enquêtés ainsi que les ressortissants de l'Europe centrale en Europe de l'ouest sont originaires en grande majorité de zones urbaines et présentaient originellement des profils d'emplois variés. Il y a parmi eux de nombreux enseignants, des membres de la police et de l'armée, de l'administration publique, des médecins, etc. Ces catégories sont moins susceptibles d'émigrer durablement. Peu de migrants expriment le désir de quitter leur pays pour toujours. «Travailler là-bas et vivre ici » était désir de vie le plus fréquemment avancé. Ces résultats sont similaires à ceux de Korczynska (1997a et 1997b) et Domaradzka (1997) sur les migrants de l'Europe centrale et de la CEI.

Une mobilité intense

LEurope centrale a été une région ayant une forte mobilité 63 millions d'entrants et 24 millions de sortants en 1989. Ces chiffres étaient supérieurs aux chiffres de la population (62 millions de personnes). En 1989, après la suppression des visas, 250 millions de voyageurs entrants et 127 millions de voyageurs sortants ont traversé les frontières des quatre pays (Hongrie, Pologne, Slovaquie, Tchéquie, WTO 2001, CESTAT 2001). Il est vrai que les distances sont courtes, et qu'un voyageur peut facilement franchir trois ou quatre frontières dans la même journée. Certaines régions sont 
peuplées par des minorités nationales, dont le pays d'origine se trouvait de l'autre côté des frontières. La visite d'amis ou de membres de la famille sont alors très fréquentes. Bien sûr, les déplacements liés au tourisme sont également populaires. La région offre une grande variété d'attractions touristiques les grandes villes disposant d'un riche héritage culturel (Prague, Budapest) font partie des destinations mondiales les plus prisées. Cependant, les achats restent un motif de déplacement important. Dans notre enquête, nous avons abordé ces déplacements en nous centrant sur la "microéconomie familiale ", leur rôle sur les dépenses de la famille selon les régions et les groupes sociaux. Nous avons ainsi pu mettre en valeur le contrastes entre deux catégories de "touristes consommateurs, les urbains aisés de Bratislava qui recherchaient en Autriche un meilleur choix et une meilleure qualité, les jeunes familles plus modestes venues souvent de régions agricoles, attirés par les faibles prix des marchés en plein air hongrois.

Le rôle des marchés en plein air et du shopping transfrontaliers en Europe centrale a changé au fil du temps. L'arrivée sur le marché d'importantes chaînes internationales de vente au détail a fortement concurrencé les marchés en plein air. Après 1993, des "nouveaux" consommateurs ont préféré faire leurs achats dans des structures commerciales, avec des prix fixes et une garantie sur les produits. Au cours des premières années du XxI ${ }^{e}$ siècle, les économies de transition d'Europe centrale se sont intensivement préparées à devenir membres de l'Union Européenne et ont rapproché leurs principales politiques fiscales, douanières et économiques des standards européens. Ce rapprochement a favorisé l'effacement des différences de prix entre les pays de la région.

Conclusion

75 L'élargissement de l'Union Européenne donne à l'Europe centrale l'occasion de rejoindre les économies capitalistes. Les exemples du Portugal et de l'Irlande indiquent que les différences majeures dans le niveau de vie peuvent diminuer sur une période de quinze à vingt ans. Le niveau de vie en Europe centrale s'approchant de celui de Europe de l'ouest, la migration de main-d'œuvre sur le modèle "néo-classique » pourrait disparaître aussi rapidement que la migration à partir du Portugal ou de l'Espagne dans les années soixante-dix et quatre-vingts. Il pourrait en aller de même pour les « achats transfrontaliers » liés à la microéconomie familiale.

La situation, aux frontières Est de la Slovaquie, la Pologne et la Hongrie, apparaît moins clairement. L'Ukraine en particulier a peu avancé dans l'introduction de réformes. En 2001, le pays a un niveau de vie faible, une économie défaillante et une situation politique en plein désarroi. L'élargissement planifié de l'Union Européenne se traduit par des projets de frontières du type Schengen entre l'Ukraine et la Pologne, la Hongrie et la Slovaquie. La Pologne et la Hongrie cherchent à protéger les minorités ethniques vivant en Ukraine, et il en découle des politiques prudentes. Les deux pays considèrent la coopération économique avec l'Ukraine comme importante, et ils ont maintenu des réglementations souples aux frontières communes. Par contre, les Républiques Tchèque et Slovaque craignent que l'afflux de main-d'œuvre migrante ukrainienne puisse mettre en danger la stabilité des marchés du travail locaux et ont introduit dès l'été 2000 des conditions assez drastiques pour l'obtention d'un visa par les Ukrainiens. Pendant un temps, cette décision politique a contribué à une diminution du nombre de commerçants, mais son impact réel sur les marchés du travail et de la contrebande a été moins important qu'escompté. La migration illégale de main-d'œuvre, le trafic et la 
contrebande sont tombés aux mains d'organisations criminelles capables de contourner la plupart des barrières officielles. Cependant, la réglementation des visas a rendu la situation plus difficile encore pour les travailleurs immigrés ukrainiens; elle les a plongés dans l'ombre et dans l'économie parallèle.

\section{BIBLIOGRAPHIE}

ALTVATER E. (1998) Theoretical Deliberations on Time and Space in Post-Socialist Transformation, Regional Studies, vol. 32, n 7, pp. 591-605.

BAUER T. (1995): The Migration Decisions with Uncertain Costs, Münchener Wirtschaftswissenschaftliche Beiträge, $\mathrm{n}^{\circ}$ 95-25, Munich.

BÖRÖCZ J. (1996) Leisure Migration: a Sociological Study of Tourism, Oxford: Pergamon.

CESTAT (2001) Statistical Bulletin, a joint publication by the Czech Statistical office, Hungarian Central Statistical Office, Central Statistical office of Poland, Statistical Office of the Republic Slovenia and Statistical Office of the Slovak Republic; Praha, Budapest, Warsaw, Ljubljana and Bratislava, 2001

CZAKÓ Á. and SIK E. (1999) Characteristics and origins of the Comecon open-air market in Hungary, International Journal of Urban and Regional Research, 23.4, pp. 715-37.

DOMARADZKA E. (1997) Incomer from East to Poland. A report on Surveys carried out in the Years 1995-1996, the Economic and Labour Market Development and International Migration - Czech Republic, Germany, Poland - joint research project, with support of the EU-Commission, working paper No. Pl-22-e/ September 1997; Institute for Employment Research - IAB Nürnberg; Germany, Institute of Labour and Social Studies - IPISS Warsaw, Poland and Research Institute of Labour and Social Affairs - VUPSV, Prague, Czech Republic.

FASSMANN H., KOHLBACHER J. and REEGER U. (1995): Die 'Neue Zuwandernung aus Ostmitteleuropa' - Eine Empirische Analyse am Beispiel der Polen in Österreich (ISR Forschungsberichte, Heft 13, Institut für Stadt and Regionalforschung Österreichische Akademie der Wischenschaften, Vienna).

FREEMAN G. P. (1986) Migration and the Political Economy of the Welfare State, Annals of the American Academy of Political and Social Science, vol. 485, pp. 51-63.

GfK (1998) Cizinci k nám jezdí nakupovat \{The GfK agency (1998): Foreigners are coming for shopping\}, Hospodáské noviny daily, $25^{\text {th }}$ February 1998, Praha.

HOFFMANN-NOWOTNY H. J. (1981), A Sociological Approach Towards General Theory of Migration, in: Kritz, M. M., Keely, Ch. B. and Tomasi, S. M. (eds.), Global Trends in Migration: Theory and Research on International Population Movements, The Centre of Migration Studies, New York.

HORÁLEK M., KOŠ M. and HORÁKOVÁ M., (1996), Work Stays of Czech Nationals in Germany, (Economic and Labour Market Development and International Migration joint research project, Working Paper No. Cz-3-e, Institute for Employment Research - IAB Nürnberg, Institute of Labour and Social Studies - IPISS Warsaw, and Research Institute of Labour and Social Affairs - VUPSV, Prague). 
IGLICKA K. (1999) The Economies of Petty Trade on the Eastern Polish Border, in: Iglicka, K. and Sword, K. (eds.): The Challenge of the East-West Migration for Poland, Macmillan Press LTD, in association with the School of Slavonic and East European Studies, London, pp. 120-144.

JERCZYNSKI M. (1999), Patterns of Spatial Mobility of Citizens of the Former Soviet Union, in: Iglicka, K. and Sword, K. (eds.) The Challenge of the East-West Migration for Poland, Macmillan Press LTD, in association with the School of Slavonic and East European Studies, London, pp. 105-119.

KOLLÁR D. (1998) Development of the Slovak labour migration and migration to Austria, in Collective of Authors Ruchliwosc prezsrezcionni ludnosci w okresie przemian ustrojowich, Universitet Mikolaja Kopernika, Torun, Poland, pp. 124-141.

KORCZYNSKA, J. (1997a) Commuting Polish Workers at the Border to Germany - Survey Results, the Economic and Labour Market Development and International Migration - Czech Republic, Germany, Poland - joint research project, with support of the EU-Commission, working paper No. Pl-26-e/ October 1997; Institute for Employment Research - IAB Nürnberg; Germany, Institute of Labour and Social Studies - IPISS Warsaw, Poland and Research Institute of Labour and Social Affairs VUPSV, Prague, Czech Republic.

KORCZYNSKA J. (1997b) Seasonal Labour Migration to Germany - Survey Results, the Economic and Labour Market Development and International Migration - Czech Republic, Germany, Poland - joint research project, with support of the EU-Commission, working paper No. Pl-27-e/ October 1997; Institute for Employment Research - IAB Nürnberg, Germany, Institute of Labour and Social Studies - IPISS Warsaw, Poland and Research Institute of Labour and Social Affairs - VUPSV, Prague, Czech Republic.

LEE E. S. (1966) A Theory of Migration, Demography (Chicago, Illinois), vol. 3., n 1, pp. 45-47.

MASSEY D. (1984) Spatial Divisions of Labour: Social Structures and the Geography of Production, London: Macmillan.

MHSR, Ministerstvo hospodárstva Slovenskej republiky (2000): Národn'y program rozvoja cestovného ruchu $v$ SR \{Ministry of Economy of the Slovak Republic 2000: National Tourism Development program in the Slovak Republic), MHSR Bratislava, May 2000.

MINCER J. (1978) Family Migration Decisions, Journal of Political Economy, vol. 86, pp. 769-773.

ODÉ A and VAN DER KNAPP B, (1998) Contemporary patterns of labour based migration to the Netherland, in C Gorter, P Nijkamp and J Poot eds, Crossing Borders: Regional and Urban Perspectives on International Migration, Aldershot, Ashgate, pp 203-221.

OECD (1998) The 1998 SOPEMI report, OECD, Paris, 1998.

OKOLSKI M. (1998) Recent Trends in International Migration - Poland 1997, Warsaw, University of Warsaw, Working Paper 19.

SIK E. and WALLACE C. (1999) The development of open-air markets in East-Central Europe, International Journal of Urban and Regional Research, vol. 23.4, pp. 697-714.

SJAASTAD L.A. (1962) The Costs and Returns of Human Migration, The Journal of Political Economy, vol. 70, pp. 80-93.

STARK O. (1991) The Migration of Labour, Basil Blackwell, Cambridge.

STORPER M. and WALKER R. (1989) The Capitalist Imperative - Territory, Technology and Industrial Growth, Oxford: Blackwell.

SWORD K. (1999), Cross-Border 'Suitcase Trade' and the Role of Foreigners in Polish Informal Markets, in Iglicka K. and Sword K. (eds.) The Challenge of the East-West Migration for Poland, 
Macmillan Press LTD, in association with the School of Slavonic and East European Studies, London 1999, pp. 145-167.

THUEN T. (1999) The Significance of Borders in the East European Transition, International Journal of Urban and Regional Research, 23.4, pp. 738-50.

TODARO M.P. (1980) International Migration in Developing Countries: A Survey, in: R.A. Easterlin ed. Population and Economic Change in Developing Countries, Chicago, NBER, pp. 361-402

ÚSTAV TURIZMU (1997) Aktívny zahranin'y cestovn'y ruch na Slovensku v roku 1996 \{Tourism Institute (1997): Incomig tourism in Slovakia in 1996\}, Bratislava 1997.

WALLACE C. (1998), Poverty and Migration in East Central Europe, Working Paper, Sociology Series, Institute for Advanced Studies, Vienna.

WALLACE C., CHMOULIAR O. and SIDORENKO E. (1996): The Eastern frontier of western Europe: mobility in the buffer zone, New Community vol. 22.2, pp. 259-286.

WALLACE C., SHMULYAR O., BEDZIR V. (1999): Investing in social capital: the case of small-scale, cross-border traders in post-Communist Central Europe, International Journal of Urban and Regional Research, 23.4, pp.751-70.

WILLIAMS A.M. and SHAW G. (1998) Western European tourism in perspective', in A.M. Williams and G.Shaw (eds.), Tourism and Economic Development: European Experiences, Chichester: Wileys.

WILLIAMS A.M. and BALÁ V. (2000) Tourism in Transition: Economic Change in Central Europe, IB Tauris, London and New York.

WILLIAMS A.M. and BALÁ V. (2001) From Collective Provision to Commodification of Tourism?, Annals of Tourism Research, vol. 28, n 1/2001 pp. 27-49.

WORLD BANK (1999) World Development Indicators, website: http: //www.worldbank.org /html/ extdr/data.htm

WORLD TOURISM ORGANISATION (1990-2001) Compendium of tourism statistics, various issues, WTO, Madrid.

ZIMMERMANN K. F. (1994) European Migration: Push and Pull, Proceedings of the World Bank Annual Conference on Development Economics 1994, Supplement to the World Bank Economic Review and the World Bank Research Observer, pp. 313-342.

\section{NOTES}

1. - Cette recherche a reçu le soutien de la Fondation Leverhulme, allocation No. F/144/ AP (UK) sur la mobilité en Europe centrale.

2. $-19 \%$ des commerçants interrogés dans cette enquête voulaient s'établir de façon permanente dans le pays dans lequel ils exerçaient leur activité. 


\section{RÉSUMÉS}

L'Europe centrale a toujours été le lieu d'importantes mobilités, mais celles-ci ont augmenté durant la période de transition au capitalisme. Cet article analyse la nature des mobilités transfrontalières (migration de travail, commerce et tourisme) et les illustre à partir d'enquêtes empiriques. Ces travaux mettent en évidence des modèles différents de mobilité selon les systèmes socio-économiques. Par exemple, les migrants d'Europe centrale se dirigeant vers les marchés du travail de l'Union Européenne étaient moins déqualifiés et avaient des comportements d'épargne différents de ceux des migrants de la CEI en Europe centrale. Les petits commerçants transfrontaliers, ressortissants de la CEI, avaient des origines sociales, des motivations et des comportements d'épargne comparables à ceux de la main-d'œuvre migrante. Le commerce transfrontalier peut donc être considéré comme une sous-catégorie de la migration de travail sur le court terme. L'article analyse aussi le tourisme transfrontalier. Les auteurs montrent qu'il existe des différences dans les motivations des acheteurs, selon qu'ils viennent de régions où les niveaux de vie sont élevés ou de régions plus pauvres. Les premiers recherchent la diversité et la qualité des produits achetés à l'étranger, les seconds une économie sur les achats, notamment dans le cas des jeunes couples.

International Mobility in Central Europe: Tourists, Traders and Migrants. -- Central Europe has been typical for a high volume of international travel, which further increased during transition period. This paper analyses particular kinds of cross-border mobility (labour migration, petty trading and tourism) and exemplifies these via a number of authors' surveys. The surveys found great differences in cross-border mobility patterns generated in different socio-economic systems. The Central Europe migrants heading for the European Union labour markets, for example, had significantly lower deskilling levels and accounted for different saving patterns than the Community of Independent Migrants in Central Europe. As for the cross-border petty trading by the CIS nationals in Central Europe, the traders had similar social backgrounds, motives and saving patterns as the labour migrants. Cross-border trading can be considered a subtype of short-term labour migration. The third part of the paper analyses shopping tourism and finds two different motives for cross-border shopping. The first one originates in regions with higher living standards and is concerned with a better assortment and/or higher-quality goods abroad. The latter one is generated via price differences, originates in poorer regions and is related to survival strategies of young families.

La movilidad internacional en Europa Central turistas, comerciantes y migrantes. -- Aunque Europa Central siempre haya sido un espacio de importante movilidad, esta ha aumentado notablemente durante el periodo de transición al capitalismo. En este artículo se analizan los tipos de movilidad transfronteriza (migración de trabajo, comercio y turismo), ilustrándolos mediante encuestas empíricas. Tales investigaciones ponen de relieve que los modelos de movilidad difieren según los sistemas socioeconómicos. Por ejemplo, los migrantes de Europa Central que se dirigían hacia los mercados de trabajo de la Unión Europea rara vez ocupaban empleos que no respetaban su nivel de cualificaron, lo que era corriente para los migrantes de la CEI en Europa Central. Sus actitudes de ahorro eran tambien diferentes. Los pequeños comerciantes transfronterizos naturales de la CEI tenían orígenes sociales, motivaciones y actitudes de ahorro comparables a los de la mano de obra migrante. En consecuencia, podemos considerar el comercio transfronterizo como una subcategoría de la migración laboral a corto plazo. En este artículo se analiza también el turismo transfronterizo. Los autores demuestran que los compradores acuden movidos por motivaciones diferentes según procedan de regiones con alto nivel de vida o de lugares más pobres. Los primeros se interesan por la diversidad y la 
calidad de los productos extranjeros, mientras que los segundos buscan compras más baratas, especialmente cuando se trata de parejas jóvenes.

INDEX

Mots-clés : frontière, enquête, travail, commerçants, tourisme, mobilité, économie

Index géographique: Union Européenne, Europe centrale

\section{AUTEURS}

ALLAN M. WILLIAMS

Department of Geography, University of Exeter, Amory Building Rennes Drive, Exeter EX4 4RJ UK

\section{VLADIMÍR BALÁ}

A.M.Williams@exeter.ac.uk 